\title{
C57BL/10 Mouse
}

National Cancer Institute

\section{Source}

National Cancer Institute. C57BL/10 Mouse. NCI Thesaurus. Code C37376.

Derived by Little (1921) from A Lathrop stocks and separated out before 1937. The C57BL/10 strain carries a Y chromosome of Asian Mus musculus orig in and a LINE-1 element from Mus spretus, suggesting that up to $6.5 \%$ of the genome is Mus spretus in origin. Substrain C57BL/10 differs from other substrains at multiple loci, including H9, Igh2 and Lv, on chromosome 4 and has a high incidence of spontaneous mutations. The mouse model has a 30\% spontaneous tumor formation in both sexes, these tumors are comprised mostly of lymphomas. The C57BL/10 strain exhibits good reproductive performance. 